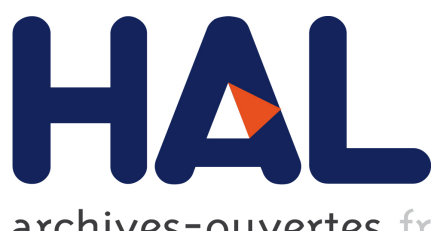

archives-ouvertes

\title{
X-ray diffraction structure measurements
}

Chrystèle Sanloup, Charlotte de Grouchy

\section{To cite this version:}

Chrystèle Sanloup, Charlotte de Grouchy. X-ray diffraction structure measurements. Magmas under pressure, 2018. hal-01867312

\section{HAL Id: hal-01867312 \\ https://hal.sorbonne-universite.fr/hal-01867312}

Submitted on 4 Sep 2018

HAL is a multi-disciplinary open access archive for the deposit and dissemination of scientific research documents, whether they are published or not. The documents may come from teaching and research institutions in France or abroad, or from public or private research centers.
L'archive ouverte pluridisciplinaire HAL, est destinée au dépôt et à la diffusion de documents scientifiques de niveau recherche, publiés ou non, émanant des établissements d'enseignement et de recherche français ou étrangers, des laboratoires publics ou privés. 
Keywords: melts structure; compression mechanisms; EDX; ADX 


\title{
Chapter 6 - X-ray diffraction structure measurements
}

\author{
Chrystèle Sanloup $^{1}$ and Charlotte J. L. de Grouchy ${ }^{2}$ \\ ${ }^{1}$ Sorbonne Universités, UPMC Univ Paris 06, CNRS, Institut des Sciences de la Terre de Paris \\ (ISTeP), 75005 Paris, France \\ ${ }^{2}$ Centre for Science at Extreme Conditions and School of Physics and Astronomy, University of \\ Edinburgh, EH9 3FD, UK. *Corresponding author (chrystele.sanloup@upmc.fr).
}

\begin{abstract}
This chapter describes how X-ray structural measurements can be done on molten silicates under high pressures, using either large volume presses or diamond-anvil cells, the latter combined with resistive heating or laser heating techniques. A brief summary of the data obtained so far is given, followed by a description of both energy-dispersive and angle-dispersive techniques, including challenges and how they may be overcome. Three areas of research are then highlighted: 1) structural measurements at extreme pressure conditions up to $100 \mathrm{GPa}, 2)$ tracking the structural environment of minor/trace elements in magmas, and 3) the different ways to obtain the density of melts from X-ray diffraction data. Finally, some future prospects are discussed.
\end{abstract}


up to $6 \mathrm{GPa}$ (Funamori et al., 2004). It demonstrated that the most important structural change in this $P$-range affects the network structure. Just above ten papers have been published since, mostly using large volume presses and energy dispersive X-ray diffraction (EDX). Processing X-ray diffraction liquid data has indeed long been considered arduous. However, the technique is now well-established and stuctural data may now be routinely collected on user-friendly synchrotron beamlines, often providing data processing softwares that they developed. In contrast, diamond-anvil cells have barely been used yet for melt structural measurements. The structure of $\mathrm{SiO}_{2}$ glass and the evolution of the Si-O coordination number were first tracked under $P$ in 1992 (Meade et al., 1992) using angle-dispersive X-ray diffraction (ADX), results corroborated later with EDX data collected up to 100 GPa over a larger $q$-range (Sato and Funamori, 2008). For DAC studies on liquids, we only cite here a few benchmark papers on with the first reports on water and Ar up to 2 GPa (Eggert et al., 2002), molten Fe up to $60 \mathrm{GPa}$ ?, and silicate melts up to $60 \mathrm{GPa}$ (Sanloup et al., 2013a). In terms of chemical composition, have been investigated mostly end-member compositions, i.e. $\mathrm{MgSiO}_{3}$ and $\mathrm{CaSiO}_{3}$ (Funamori et al., 2004), $\mathrm{NaAlSi}_{3} \mathrm{O}_{8}$ (Yamada et al., 2011), $\mathrm{NaAlSi}_{2} \mathrm{O}_{6}$ (Sakamaki et al., 2012), $\mathrm{CaMgSi}_{2} \mathrm{O}_{6}$ (Wang et al., 2014), $\mathrm{Fe}_{2} \mathrm{SiO}_{4}$ (Sanloup et al., 2013b), $\mathrm{MgSiO}_{3}-\mathrm{Mg}_{2} \mathrm{SiO}_{4}$ both dry and hydrous (Yamada et al., 2007, 2011), and only two more complex compositions, i.e. basalt (Sakamaki et al., 2013; Sanloup et al., 2013a; ?) and hydrous haplogranite (Anderson et al., 2014; ?). The choice of simple end-member compositions is guided by the fact that unlike with X-ray absorption techniques that are chemically selective, all ion-ion distribution functions contribute to the X-ray diffraction signal, hence simple end-member are necessary to identify individual contributions. That said, considerable improvements in the technique now allow to study some minor but key geochemical elements (?), such as heavy rare Earth's, and relate changes in their structural environment in melts to changes in their partitioning behaviour (mineral/melt or metal/melt). One can note that pure $\mathrm{SiO}_{2}$ melt still remains a challenge due to the highest $T$ involved. This chapter will first describe the experimental techniques, with a brief account on large-volume press data (readers are referred to Chapter by Sakamaki for results obtained using this technique), and more details on how to collect X-ray diffraction data on silicate melts using diamond-anvil cells combined with either resistive heating or laser heating. Secondly, some results will be presented such as the highest current $P-T$ data on melts, probing the local environment of minor elements, monitoring density evolution while tracking 
changes in the intermediate-range order. Finally, some challenges and future directions will be highlighted.

\section{Experimental techniques}

\subsection{From X-ray diffraction data to structural information}

The measured experimental intensity, $I(q)$, contains scattering by both the sample and background contributions, where $q=\frac{4 \pi}{\lambda} \sin \theta$ and $\lambda$ is the x-ray beam wavelength. In order to isolate the intensity that contains only the structural information, corrections for background intensity, $I_{B}(q)$, and attenuation, $A(q)$, from the sample must be made to the measured intensity given by

$$
I(q)=I_{s}(q)+A(q) I_{B}(q)+I_{i n c}(q),
$$

where $I_{s}(q)$ represents the scattering from the sample containing only structural information, and $I_{\text {inc }}(q)$ represents the incoherent scattering from the atoms, arising from the sum of the self $\sum_{\alpha} c_{\alpha} f_{\alpha}(q)^{2}$ and Compton scattering $\sum_{\alpha} c_{\alpha} C_{\alpha}(q)$, where $c_{\alpha}$ is the concentration of species $\alpha$. The values for the self and Compton scattering amplitudes are documented by Hajdu (1972); Hubbell et al. (1975). The Faber-Ziman total structure factor, $S(q)$, (Faber and Ziman (1965)) is then determined by;

$$
S(q)-1=\frac{K\left[I_{s}(q)-A(q) I_{B}(q)\right]-I_{i n c}(q)}{\left\langle f^{2}(q)\right\rangle},
$$

where $K$ is required to normalise the data to the incoherent scattering profile and the total structure factor is normalised to the average scattering $\left\langle f^{2}(q)\right\rangle=\left(\sum_{\alpha} c_{\alpha} f_{\alpha}(q)\right)^{2}$. The radial distribution functions, $G(r)$, are then obtained from a Fourier transform of the $S(q)$ as:

$$
G(r)-1=\frac{1}{2 \pi^{2} r n_{0}} \int_{0}^{\infty} q[S(q)-1] \sin (q r) \mathrm{dq},
$$

where $n_{0}$ is the atomic density in atoms per $\AA^{3}$.

The technical challenges here are three-fold: 1$)$ to reach high $T$ conditions under high $P$, silicates being refractory materials with high melting points, 2 ) to have sufficiently large sample volume probed by the X-rays, silicate melts being amorphous by nature and with relatively low mean atomic number (circa 20-21) are consequently very weak scatterers, and 3) to collect diffraction data on the largest $q$ range possible as the 
structural information contained in the radial distribution function, $g(r)$, is obtained by Fourier transforming the normalised intensity, i.e. the structure factor, $S(q)$ (cf. equation 3).

\subsection{Large-volume presses}

By far the most used method, the energy-dispersive diffraction remains unrivalled in terms of accessible $q$-range on compressed amorphous samples $\left(15 \AA^{-1}\right.$ routinely, occasionally higher), and consequent spatial resolution in real space. The maximum $P$ range however is still modest, up to $8 \mathrm{GPa}$ maximum, as sample volume must be optimised.

Energy-dispersive diffraction (EDX) set-up. EDX is used with multi-anvil presses due to the limited angular access to the sample (unless using transparent anvils made of diamond or cBN, but this has not been done yet to study melts). Not that many changes in the technique, except the use in conjonction with the Paris-Edinburgh cell that necessitated efforts to adapt the cell-assembly but with the advantage of a larger angular access to the sample and therefore much increased $q$-range (up to $22 \AA^{-1}$ vs $15 \AA^{-1}$ with a multi-anvil press. Other advantage of PE-EDX: higher signal/noise ratio as transparent materials can be inserted along the X-ray paths in a stable way Yamada et al. (2011). Measurements are taken at fixed detector $2 \theta$ angle (typically ten angles ranging between $2^{\circ}$ and $35^{\circ}$ ). This covers up to $20 \AA^{-1}$ in reciprocal space where $q=4 E \sin (\theta) / 12.398$ where $E$ is energy in keV of the X-rays up to $120 \mathrm{keV}$. Background signal from the sample cell environment is geometrically filtered by the intersection between incident beam and detector angle (collimator). Unknowns are the white beam profile and $\mathrm{xx}$, and can be obtained from the highest angle pattern, assuming that the diffuse signal from the molten sample is negligible at such high $q$ values (refer to C. Park manual). Alternatively, these parameters can be obtained by fitting the baselines on a crystalline data set ?. Peaks in the diffraction pattern may arise from Bragg peaks of the sample container (usually graphite, more rarely diamond) and fluorescence from indium on the detector and elements within the sample; peaks must all be removed. The scaling of the individual structure factors is achieved by fixing the oscillations of the data at highest $q$ to oscillate around 1 as they must by definition. Then the other structure factors are scaled accordingly in reverse order from highest $q$. After merging, an error weighted spline is fit to the data in order to obtain an evenly spaced $S(q)$ distribution. This fitting uses only parts of the individual $S(q)$ 
that overlap in order that they can be fit together. More details on how to process EDX data on melts may be found in K-i Funakoshi 1997 (book chapter?). Note that EDX may also be used in conjunction with diamond-anvil cells, as illustrated for example by the structure of $\mathrm{SiO}_{2}$ glass obtained up to $100 \mathrm{GPa}$ (Sato and Funamori, 2010), so in principle this technique could be used for the study of melts as well. The main problem being the acquisition time (not given in SatoFunamori papers) of typically a few hours, a duration over which it is very challenging to maintain stable high $T$ conditions in a diamond-anvil cell.

Angle-dispersive diffraction (ADX) set-up. ADX necessitates a large angular access to the sample, which is possible if using a device using two opposed anvils such as the ParisEdinburgh press. Background removal by either subtracting pattern collected outside of the sample, or by filtering the signal using multichannel collector that acts similarly to the collimating slits with EDX. The latter efficiently removes the background (except at low angles), but looses efficiency at high energies as it becomes more transparent to the X-rays. Currently, molten basalt data collected up to $8 \AA^{-1}$ using this technique (Crépisson et al., 2014), although up to $18 \AA^{-1}$ was achieved on molten Fe (Sanloup et al., 2000) using high energy X-rays $(101.7 \mathrm{keV})$ without using a collimation system, which might remain necessary for low Z silicate melts. Collection times: 10-30 minutes.

\subsection{Diamond-anvil cells}

As mentioned above, only ADX set-ups have been used so far with diamond-anvil cells for the structure of molten silicates. DAC are very versatile tools and the most straightforward way to cover all $P-T$ conditions relevant for planetary interiors. As for any ADX-based high $P$ set-up, it is crucial to optimize the angular access to the sample that is necessarily restricted by the high $P$ environment. Efforts to increase the accessible $q$-range have used transparent seats (beryllium or BN in Shen et al for Fe, + modified gaskets with transparent materials around the sample, check Eggert). The availability of Boehler-Almax anvils (ref) and adapted large-opening WC seats (70 or $80^{\circ}$ ) have largely helped to democratise the technique, and are now the simplest way now to optimize the accessible $q$-range while also reducing Compton inelastic scattering from the diamonds as BA anvils are thinner than conventional ones $(1.5 \mathrm{~mm}$ thickness vs 2.0-3.0 mm). The elastic contribution of diamond anvils to the signal, i.e. Bragg peaks, may be minimized by rotating the DAC perpendicularly to the X-ray beam to avoid the most saturating peaks (Fig.1). All diamond Bragg peaks must then be removed 
using masks. In DAC experiments, a very thin sample (maximum few tens of microns, often only a few microns thick) is squeezed between two diamond anvils (1.5 to $2 \mathrm{~mm}$ each). As a result, the largest contribution to the signal is the inelastic scattering from the diamonds. It must be precisely measured and removed from the total intensity in order to extract the sample signal. For this purpose, one can measure the empty cell background, i.e. remove the sample after the experiment, put the empty gasket back in place and collect XRD data. Ideally, the background may also be obtained from the crystalline pattern, after quenching the experiment to room $T$, and after removing all Bragg peaks from the integrated intensity. Silicate melts are often complex chemical compositions and crystallise as forest of peaks that cannot be individually removed. It is nonetheless helpful to scale the empty cell background up to the baseline. For further description of background removal methods, readers may refer for ADX data to Eggert et al. (2002) and ? (Rev Sci Instr), and for EDX data to Sato and Funamori (2010).

Resistive-heating diamond-anvil cells (RH-DACs). Due to their relatively low melting points, only hydrous continental crust magmas may be studied using resistive heating techniques (maximum achievable $T$ of $1300 \mathrm{~K}$ with metallic wires, up to $1800 \mathrm{~K}$ using graphite furnace in conjunction with a vacuum chamber, see Liermann et al. for a review of resistive heating techniques); this is nonetheless a large and important field of study. The advantages of RH-DAC are two-fold: good control on $T$ and relatively large sample volume as no thermal insulation is required, therefore the gasket hole can be completely filled with the sample. On the relatively small $P$ range over which continental crust magmas are molten below $1300 \mathrm{~K}$, a large beam size is not an issue as one can use 250-300 micronm sample chambers; therefore the sample volume probed by the X-rays may also be enlarged by using a relatively large beam. The haplogranite (HPG) data shown on Figures 2 were collected on relatively thick samples for DAC experiments, 120 micronm, and using X-ray beam energy ranging from $30 \mathrm{keV}$ to 60 $\mathrm{keV}$. Increasing the energy is essential to increase the $q$-range, with the draw-back that the beam intensity is usually highest in the $20-30 \mathrm{keV}$ range and rapidly drops at higher energies(?). Comparison of data collected on the same HPG sample using different energies (Fig.2) shows that a good compromise between photon flux and $q$ range is reached at $47 \mathrm{keV}$. A $60 \mathrm{keV}$ or higher energy set-up is necessary for the study of some minor/trace elements which contribution on $g(r)$ would otherwise overlap too much with other ion-ion contributions, but it necessitates much longer acquisition times.

High energy X-ray diffraction data may be collected on synchrotron beamlines not 
IPs.png

Figure 1: 2-D diffraction data collected on molten hydrous haplogranite. Right panel: at $33 \mathrm{keV}$ on a MAR555 (ID09, ESRF); middle panel: at $42 \mathrm{keV}$ on a MAR3450 (I15, Diamond); left panel: same as middle panel with masks for diamond Bragg peaks. NB: the strong inner diffuse ring is the first sharp diffraction peak. 


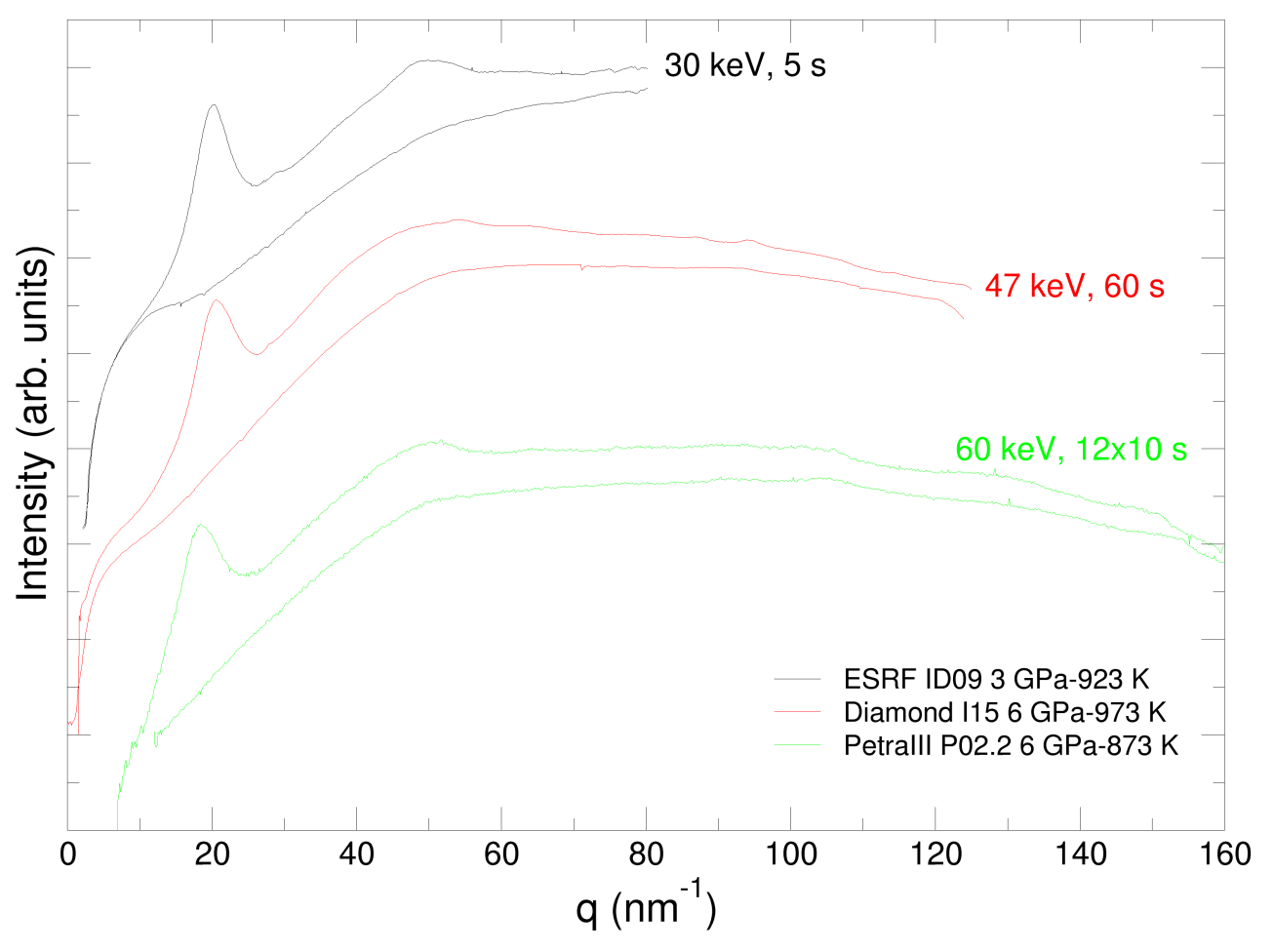

Figure 2: Integrated intensity on HPG melt $\left(T>T_{g}\right)$ and corresponding empty cell background using similar RH-DAC but different X-ray wavelength. Top (black curves): at $30 \mathrm{keV}$, data collected on ID09 at the ESRF for 5 seconds, $2 \mathrm{xX}$ micronm beam size; middle (red curves): at $47 \mathrm{keV}$, data collected on I15 in Diamond for 60 seconds, 20 micronm beam size; bottom (green curves): at $60 \mathrm{keV}$, data collected on P02.2 in PetraIII-DESY, 4micronm x6 micronm beam size. 

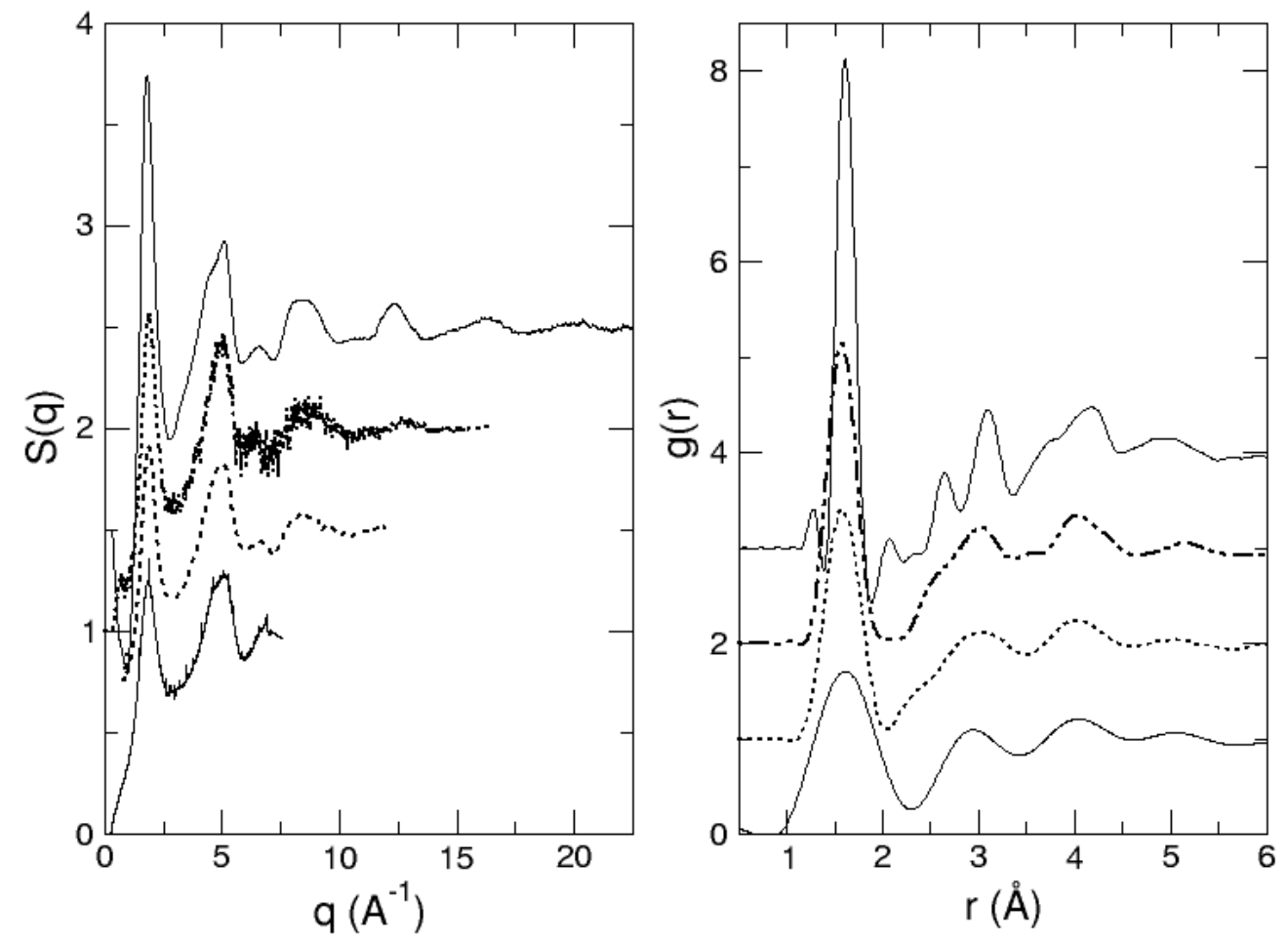

Figure 3: Structure factor, $S(q)$, and corresponding $g(r)$. From top to bottom: 1) free standing HPG glass, data collected on I15, ESRF at $72.9 \mathrm{keV}$ for $10 \mathrm{msec}$, 2) molten hydrous HPG at X GPa and $\mathrm{X} \mathrm{K}$, data collected at $60 \mathrm{keV}$ (PetraIII-DESY, P02.2), 3) molten hydrous HPG at X GPa and X K, data collected at same conditions as for Fig.2. 
dedicated to high $P$ experiments for amorphous samples. However, for combined high $P$ and high $T$ experiments on melts, although it is possible for users to bring their own DAC heating set-up whether that be resistive furnaces or portable laser set-up, the use of dedicated high $P$ beamlines is recommended due to the complexity of recording X-ray structural data on melts, including precise alignment procedures on systems that may not be stable over long durations.

Laser-heating DACs. This technique has been used to obtain the structure of molten Fe up to $58 \mathrm{GPa}$ ? by directly heating the sample, or that of water up to $57 \mathrm{GPa}$ Goncharov et al. (2009) using Ir foil that couples with the laser, to conductively heat the sample. Laser-heating requires that the sample be thermally insulated from the diamonds, as they are very good heat conductors. For silicate melts Sanloup et al. (2013a), pure silica platelets proved ideal as crystalline peaks are very thin and easy to remove from the 1-D integrated diffraction pattern, and no chemical contamination was observed on recovered samples at least on the short timescale of the experiment (10 $\mathrm{s}$ heating). Laser heating in diamond anvil cells is currently most commonly achieved using a Nd-YAG laser that couples with $3 \mathrm{~d}$ elements, typically Fe which is present in most natural magmatic compositions, and can be focussed to relatively small spot size (typically $20 \mu \mathrm{m}$ ). For these experiments, flash-heating is necessary as Fe tends to move away from the heated area due to the Soret effect. Flash-heating is achieved by ramping the laser to the target power with the shutter closed, and simultaneously opening laser and X-ray shutters for data acquisition (typically $10 \mathrm{~s}$ ) before quenching. $\mathrm{CO}_{2} \mathrm{IR}$ laser can be used for Fe-free compositions, however focussing is arduous compared to YAG lasers, and only thin samples may be properly heated, thus further diminishing the signal intensity (Drewitt et al., 2015). It is preferable to use off-axis laser heating rather than on-axis laser heating as the latter is done using carbon mirrors that alter the signal significantly especially for low intensity scattering materials such as molten silicates (see kink in the signal at 20 degrees on Fig.4).

It is now possible to obtain accurate structural information on molten silicates up to the megabar range. But it remains very costly above 40-50 GPa as both diamonds get burned due to the large sample thickness (circa 30-40 micronm) necessary to obtain a sufficiently strong scattered intensity, and the consequent thinner thermal insulation layers (circa 10-15 micronm on both sides). Nd-doping might be useful in this respect. Preliminary experiments were conducted on synthetic Anorthite-Diopside composition doped with 3.8 wt.\% $\mathrm{Nd}_{2} \mathrm{O}_{3}$. The $\mathrm{Nd}$ coupler proved to have many advantages over 
previous iron coupling measurements, including the ability to remelt the sample with identical quality, very localised heating, and preservation of the diamond anvils even at $P>50 \mathrm{GPa}$ and $T>2000 \mathrm{~K}$. When Fe has been used as a coupler (Sanloup et al., 2013a), if the same sample was melted more than once with the laser a considerable decrease in intensity was observed, indicating that Fe appeared to diffuse away from the sample spot. With Nd no such decrease in intensity was observed and the recovered sample analysis suggests that $\mathrm{Nd}$ remained homogeneously distributed. The localisation of heating is improved with $\mathrm{Nd}$ compared to $\mathrm{Fe}$ as observed by the partial recrystallisation of the $\mathrm{SiO}_{2}$ thermal insulation layer. In Fe-doped samples this layer always recrystallised resulting in considerably more Bragg peaks to remove and additional uncertainty in the data processing. It is also this improvement in localisation of heating that appears to protect the diamond anvils from damage at high $P$ and $T$.

\section{Examples of the results by the technique}

Three main areas: local environment around major cations, around minor cations, and medium-range order. And density.

\subsection{Extreme P-T}

Only two studies have been published so far on the structure of silicate melts above the $10 \mathrm{GPa}$ range, one on molten basalt up to $60 \mathrm{GPa}$ (Sanloup et al., 2013a) and one on alumino-silicate melts up to $30 \mathrm{GPa}$ Drewitt et al. (2015). First aim: X-O coordination number and bond length of major elements within 2-3 A max, too many correlations afterwards and too much disorder. Coordination number vs $\mathrm{P}$ established now for Fe, $\mathrm{Si}$, and $\mathrm{Al}$ at least on some P-range, all show increase of $\mathrm{X}-\mathrm{O}$ over a given P-range (i.e. continuous transition). Remain to be measured in the melt: $\mathrm{Mg}$ (done in glass), $\mathrm{Ca}$ (difficult due to broad distribution). These data-sets provide a physical ground for the understanding of compression mechanisms in melts (Sanloup, 2016). Brief summary of Nature paper (Sanloup et al., 2013a). The $P$-range has now been pushed further, with acquisition of data up to $104 \mathrm{GPa}$ (Fig.6) at which point both lasers were at their maximum power of $100 \mathrm{~W}$ each which prevented the acquisition of data at higher $P$, i.e. with higher melting points. The level of intensity is similar to data collected up to $60 \mathrm{GPa}$, and should provide quantitative structural information once processed (results will be published elsewhere). We should then be able to test whether or not Si-O increases further than 6 as proposed for silicate glasses based on acoustic velocity 
Nd_AnDio.png

Figure 4: Molten anorthite-diopside at $3500 \mathrm{~K}(10 \mathrm{~s})$ and quenched glass $(60 \mathrm{~s})$ at $9 \mathrm{GPa}$ (data collected on GSECARS ID-13, APS at $40 \mathrm{keV}$ using a Perkin Elmer detector); note the kink in the overall patterns at 20 degrees due to scattering from the carbon window used for laser heating at 90 degrees geometry. Left panel: intensity (melt, quenched glass, empty cell); middle panel: melt (red) and glass (black) structure factor, $S(q)$; right panel: melt $g(r)$ (continuous line) and fit of the first peak using $\mathrm{CN}$ Si-O=6, CN Al-O=5.2). 


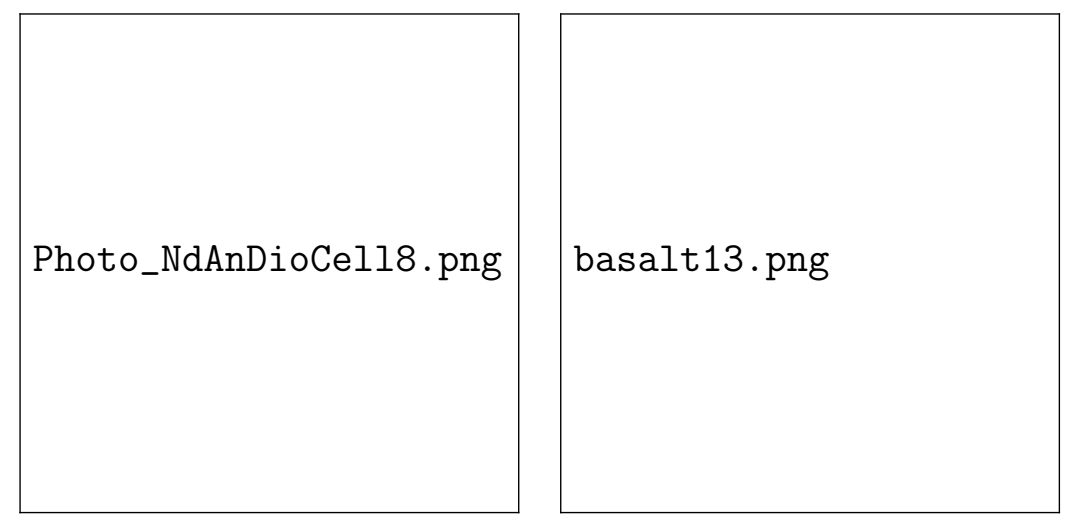

Figure 5: Microphotographs of a Nd-doped Fe-free anorthite-diopside sample after repeated laserheating at $29.3 \mathrm{GPa}$ (left), and of a basalt sample after three laser-heatings at $45 \mathrm{GPa}$ (check for run 13) (right). Quenched molten zones appear as spheres on both samples.

measurements ?, and reported for glassy $\mathrm{GeO}_{2}$ structural measurements (?). Therefore in principle, X-ray data can be collected at least over the whole megabar range on molten silicates.

\subsection{Minor elements}

Context: element partitioning depends not only on crystal structure and how it changes with $\mathrm{P}$, but also on melt composition, e.g. on melt structure. This well established fact at ambient pressure becomes more documented now under pressures. Our understanding of planetary differentiation, or any magmatism-related issue, therefore depends on our understanding of melt 'crystal-chemistry'. Complementary with X-ray absorption techniques. Limitations of XAS: only edges above $15 \mathrm{keV}$ are accessible through a high pressure apparatus, and edges above $35 \mathrm{keV}$ challenging due to low flux (check). This restrict the study to the following elements (?). Limitations of XRD: the $\mathrm{X}-\mathrm{O}$ bond must not overlap too much with major elements bonds in order to be detected with the full chemical information given by radial distribution function. Only heavy elements accessible/detectable. Proved feasible for $\mathrm{Lu}(\mathrm{Z}=71)$. Ideal window: X-O bond ranging between $\mathrm{Al}-\mathrm{O}$ and $\mathrm{Ca}-\mathrm{O}$ bond length. Easier for HPG simple composition, but also feasible for more complex An-Dio composition (?). Conclusion: trace elements may change coordination number abruptly, or at least on much smaller P-range than reported for major elements. Clearly requires larger q-range than to solve major elements local environment. Data are now coming, need proper theoretical treatment now. 
basaltUHP.png

Figure 6: Intensity scattered by molten basalt at the highest $P$ at $80 \mathrm{GPa}$ and $104 \mathrm{GPa}$, the highest $P$ investigated so far on molten silicates using static compression techniques (PetraIII-DESY, P02.2; $42 \mathrm{keV}, 10 \times 1 \mathrm{~s}$ acquisition). 


\subsection{Tracking the medium-range order/compression mechanisms}

There are several ways to obtain the density of silicate melts using X-ray diffraction. First, one can use the self-consistent method and obtain the density by minimising the signal in $g(r)$ at distances lower than the interatomic distances (see ? for the method and Eggert et al. (2002) for its adaptation to diamond-anvil cell data). Second, for low $P$ data, one may use the fact that $S(q)$ limit at low $q(?)$. From

$$
\lim _{q \rightarrow 0} S(q)=\frac{n T k_{B}}{K_{T}}
$$

and

$$
\left.K_{T}=\rho \frac{\partial P}{\partial \rho}\right)_{T}
$$

we get

$$
\left.\frac{\partial P}{\partial \rho}\right)_{T}=\frac{\mathcal{N}_{A} k_{B} T}{M S(0)}
$$

where $k_{B}$ is the Boltzmann constant, $\mathcal{N}_{A}$ is the Avogadro number, and $K_{T}$ the isothermal bulk modulus. Equation 6 is valid for any isotropic liquid (Egelstaff, 1994), independently on the nature of bonding within the melt. This second method may only be used at low $P$ as the noise on $S(q)$ gets larger than $S(0)$ for high density melts (i.e. high bulk modulus), and it is not possible to determine

$$
\lim _{q \rightarrow 0} S(q)=\frac{n T k_{B}}{K_{T}}
$$

in such conditions.

Third, one can also constrain the density from the radial distribution function if the coordination number of the main cations are known. For instance, the density, $\rho$, of the HPG was estimated by fixing the Si-O coordination number to 4 which is reasonable in this relatively low $P$ range, and fitting the first peak on $g(r)$ (give equation to see where $\mathrm{n}$ and $\mathrm{CN}$ come into play). In order to compare with reported densities in the literature, the calculated atomic number density, $n$, must be converted to $\mathrm{g} / \mathrm{cm} 3$. This is done using the formula

$$
\rho=\frac{n M}{\mathcal{N}_{A}}
$$

where $M$ is the atomic mass of the sample. The densities for the hydrous glass and melt samples are shown in Figure ?? and compared to hydrous granitic melt densities reported by Malfait et al. (2014).

Fig.: HPG density from Charlotte. 
Mention evolution of FSDP as the main compression mechanism at low $P$ for silicate melts. Including effect of water by Yamada 2007 (?). But careful not to overlap too much on Sakamaki chapter. Cite Zeidler and Salmon work. Position of FSDP equal to 2pi/distance (ring size?) cf ChemGeol 2016 and packing limit (Wang 2014).

Fig.: FSDP vs P HPG, dry and hydrous

\section{Challenge and future perspectives}

\subsection{How to further optimize the signal/diffracted intensity, and the accessible q-range/spatial} resolution in direct space

Using Soller slits on DACs (in fact not used to increase q-range that much but to get signal from low scattering materials)? May be mention and add that structure of molten water was obtained up to $57 \mathrm{GPa}$ in DAC without Soller slits, eventually comparing the signal with recent data obtained with Soller slits (Datchi: donnees sur $\mathrm{H} 2$ a tres basse pression, et donnees sur $\mathrm{CO} 2$ ). Clearly increasing the energy is a better option. Katayama 2010: up to 17 GPa Datchi PRB 2016 on molten CO2 up to 10 GPa: data collected up to 10A-1 but cut at 7.4 A-1 due to noise/signal ratio Discuss dedicated HP high energy beamlines rather than bringing DACs on high-energy beamlines due to the challenging nature of the experiments. Or rather waiting for very high $\mathrm{P}-\mathrm{T}$ in multi-anvil presses, might be the best option. What to expect from upgraded sources in terms of liquid diffraction.

\subsection{Switching from static to dynamic compression}

And most of all, shock wave data!! Shock wave data provide key constraints on the melting curve of planetary materials, including silicates (?). Liquid-liquid phase transition has been reported (?), followed by metallisation of the melt at higher pressures. First quantitative structural data on molten materials obtain on bismuth in 2015 at 14 GPa using free electron laser shock-wave experiments (?), and already getting decent signal on molten scandium at $82 \mathrm{GPa}(?)$.with the exceptional advantage that there is no background to remove, and complementary good constraints on the pressure and bulk density of the shocked sample from VISAR diagnostic. The quality of the data is increasing rapidly, and this a very promising area for the structure of liquids at high pressure in general and silicate melts in particular, with implications ranging from understanding the magma ocean area and its eventual remnants at the bottom of the lower mantle, to the formation, structure and dynamics of super Earths exoplanets. 
319

\section{Acknowledgements}

I thank my collaborators Benjamin Cochain, Clemence Leroy, Jessica Hudspeth, Helene Bureau, Burkhardt Schmidt, Veronika Afonina, and beamline contacts D. Daisenberg (Diamond, I15), Lucile Bezacier (ESRF, ID09), Z. Konopkova (PetraIII-DESY, P02.2), C. Prescher (GSECARS, APS) for their help during the experiments, and Simon A. J. Kimber (ESRF, I15) who collected the data on HPG glass at ambient pressure. This work was supported by the European Research Council under the European Community's Seventh Framework Programme (FP7/20072013 Grant Agreement No. 259649). Financial support of synchrotrons: data from GSECARS, PETRA, ESRF, Diamond. 


\section{References}

Anderson, A. J., Yan, H., Mayanovic, R. A., Solferino, G., Benmore, C. J., 2014. Highenergy X-ray diffraction of a hydrous silicate liquid under conditions of high pressure and temperature in a modified hydrothermal diamond anvil cell. High Press. Res. 34 (1), 100-109.

Crépisson, C., Morard, G., Bureau, H., Prouteau, G., Morizet, Y., Petitgirard, S., Sanloup, C., 2014. Magmas trapped at depth and the continental lithosphereasthenosphere boundary. Earth Planet. Sci. Lett. 393, 105-112.

Drewitt, J. W. E., Jahn, S., Sanloup, C., de Grouchy, C., Garbarino, G., Hennet, L., 2015. Development of chemical and topological structure in aluminosilicate liquids and glasses at high pressure. J. Phys.: Cond. Matt. 27, 105103.

Egelstaff, P. A., 1994. An Introduction to the Liquid State. Oxford University Press, Oxford.

Eggert, J. H., Weck, G., Loubeyre, P., Mezouar, M., 2002. Quantitative structure factor and density measurements of high-pressure in diamond anvil cells by x-ray diffraction: Argon and water. Phys. Rev. B 65, 174105.

Faber, T., Ziman, J., 1965. A theory of the electrical properties of liquid metals. Philos. Mag. 11, 153-173.

Funamori, N., Yamamoto, S., Yagi, T., Kikegawa, T., 2004. Exploratory studies of silicate melt structure at high pressures and temperatures by in situ X-ray diffraction. J. Geophys. Res. 109, B03203.

Goncharov, A. F., Sanloup, C., Goldman, N., Crowhurst, J. C., Bastea, S., Howard, W. M., Fried, L. E., Guignot, N., Mezouar, M., Meng, Y., 2009. Dissociative melting of ice VII at high pressure. J. Chem. Phys. 130, 124514.

Hajdu, F., 1972. Revised parameters of the analytic fits for coherent and incoherent scattered x-ray intensities of the first 36 atoms. Acta Cryst., 250-252.

Hubbell, J. H., Veigele, W. J., Briggs, E. A., Brown, R. T., Cromer, D. T., Howerton, R. J., 1975. Atomic form factors, incoherent scattering functions, and photon scattering cross sections. J. Phys. Chem. Ref. Data 4, 471. 
Malfait, W. J., Seifert, R., Petitgirard, S., Perrillat, J.-P., Mezouar, M., Ota, T., Nakamura, E., Lerch, P., Sanchez-Valle, C., 2014. Supervolcano eruptions driven by melt buoyancy in large silicic magma chambers. Nat. Geoscience 7, 122-125.

Meade, C., Hemley, R. J., Mao, H. K., 1992. High-pressure x-ray diffraction of $\mathrm{SiO}_{2}$ glass. Phys. Rev. Lett. 69, 1387-1390.

Sakamaki, T., Suzuki, A., Terasaki, H., Urakawa, S., Katayama, Y., Funakoshi, K., Hernlund, J., Ballmer, M., 2013. Ponded melt at the boundary between the lithosphere and asthenosphere. Nature Geo. 6, 1041-1044.

Sakamaki, T., Wang, Y., Park, C., Yu, T., Shen, G., 2012. Structure of jadeite melt at high pressures up to 4.9 GPa. J. Appl. Phys. 111, 112623.

Sanloup, C., JUL 1 2016. Density of magmas at depth. Chem. Geol. 429, 51-59.

Sanloup, C., Bonev, S. A., Hochlaf, M., Maynard-Casely, H. E., 2013a. Reactivity of xenon with ice at planetary conditions. Phys. Rev. Lett. 110, 265501.

Sanloup, C., Drewitt, J. W. E., Konôpková, Z., Dalladay-Simpson, P., Morton, D. M., Rai, N., van Westrenen, W., Morgenroth, W., 2013b. Structural change in molten basalt at deep mantle conditions. Nature 503, 104-107.

Sanloup, C., Guyot, F., Gillet, P., Fiquet, G., Hemley, R., Mezouar, M., Martinez, I., 2000. Structural changes in liquid Fe and Fe alloys at high pressures and high temperatures from synchrotron X-ray diffraction. In: Geophysical Research Abstracts, EGS 25th General Assembly. Vol. 2.

Sato, T., Funamori, N., 2008. Sixfold-coordinated amorphous polymorph of $\mathrm{SiO}_{2}$ under high pressure. Phys. Rev. Lett. 101, 255502.

Sato, T., Funamori, N., 2010. High-pressure structural transformation of $\mathrm{SiO}_{2}$ glass up to 100 GPa. Phys. Rev. B 82, 184102.

Wang, Y. B., Sakamaki, T., Skinner, L. B., Jing, Z., Yu, T., Kono, Y., Park, C., Shen, G., Rivers, M. L., Sutton, S. R., 2014. Atomistic insight into viscosity and density of silicate melts under pressure. Nature Comm. 5, 3241.

Yamada, A., Inoue, T., Urakawa, S., Funakoshi, K.-i., Funamori, N., Kikegawa, T., Ohfuji, H., Irifune, T., 2007. In situ X-ray experiment on the structure of hydrous 
$386 \mathrm{Mg}$-silicate melt under high pressure and high temperature. Geophys. Res. Lett. $38734(10)$.

388 Yamada, A., Wang, Y., Inoue, T., Yang, W., Park, C., Yu, T., Shen, G., 2011. High389 pressure $\mathrm{x}$-ray diffraction studies on the structure of liquid silicate using a Paris$390 \quad$ Edinburgh type large volume press. Rev. Sci. Instr. 82, 015103. 